\title{
Aplicación de la energía solar fotovoltaica interconectada a la red eléctrica. Caso de estudio Thierhaupten-Alemania-
}

\author{
Pablo Castellot Pérez* \\ Kai Edwin Whiting** \\ Fecha de recepción: 11 de agosto de 2014 \\ Fecha de aprobación: 2 de octubre de 2014 \\ Pp. $145-170$
}

\section{RESUMEN}

El presente estudio evalúa la viabilidad de una instalación solar fotovoltaica, conectada a la red para la generación de electricidad en una ubicación localizada en Thierhaupten, en la ciudad de Augsburg, al sur de Alemania. El dimensionado y el diseño del sistema se realizó mediante el uso de las herramientas: PVGis, PV-Sol, Sunny Design, Würth y hojas de cálculo Excel. Se analiza el desempeño técnico y económico de un sistema conformado por celdas fotovoltaicas y una capacidad de 78,96 kWp. El promedio de radiación solar global media anual es de $3000 \mathrm{Wh} / \mathrm{m} 2 /$ día, y por lo tanto, se proyecta una generación de 75.043 kWh/año de electricidad. Con base en el precio de la energía solar fotovoltaica, regulado por la normativa de la Ley de Energía Renovable (Erneuerbare Energien Gesetz) vigente en Alemania, que asciende a los 0,16 €/kWh, de los resultados se concluye que el proyecto estudiado es viable con un TIR (Tasa Interna de Retorno) de 15,2\% y VPN (Valor Presente Neto) de €14,966.72.

\section{Palabras Clave}

Energías renovables, energía solar fotovoltaica, interconexión eléctrica.

\footnotetext{
* Magíster en Energías Renovables y Pregrado en Ingeniería Industrial, Universidad de Zaragoza.

** Maestría en Energías Renovables, Universidad de Zaragoza; y en Ambiente y Negocios, University of Abertay Dundee. Director de la carrera de Ingeniería en Energías de la Universidad EAN.

*** Magíster en Eficiencia Energética y Ecología Industrial, Universidad de Zaragoza. Especialista en Gerencia de Proyectos y Gestión HSEQ, Universidad EAN. Ingeniero Ambiental y Sanitario, Universidad de La Salle. Facultad de Ciencias Ambientales, Universidad Piloto de Colombia.
} 


\section{Application of solar photovoltaic energy interconnected to the electrical network. Thierhaupten case study - Germany -}

\section{Abstract}

This paper describes the viability of a grid connected solar photovoltaic installation, with a capacity of $78.96 \mathrm{kWp}$ located in Thierhaupten, near Augsburg. The dimensioning and designing of this system was made by the authors using the following tools: PVGis, PV-Sol, Sunny Design and Würth, along with Microsoft Excel. The expected performance at both, technical and economic levels, was observed. The average solar radiation experienced at the site was $3000 \mathrm{Wh} / \mathrm{m} 2 /$ day, which resulted in an electricity generation of $75.043 \mathrm{kWh} /$ year. The norm established by German legislation (Erneuerbare Energien Gesetz) for renewable energy stated that the baseline price was $0.16 € / \mathrm{kWh}$, and the results showed that this project was financially viable, providing a NPV (net profit value) of $€ 14,966.72$ and a return rate of $15.2 \%$.

\section{KEY WORDS}

Renewable energies, Solar Photovoltaic installation, Grid interconnection.

\section{Énergie solaire photovoltaïque interconnectée au réseau électrique. Étude de cas de Thierhaupten (Allemagne)}

\section{RÉSUMÉ}

Cette étude évalue la faisabilité de l'installation d'un système solaire photovoltaïque connecté au réseau national pour la production d'électricité à Thierhaupten, dans la ville d'Augsbourg, au sud de l'Allemagne. La conception et les plans du système ont été réalisés grâce aux instruments PVGis, PV-Sol, Sunny Design, Würth et des tableurs Excel. Les performances techniques et économiques d'un système composé de cellules photovoltaïques d'une capacité de 78,96 kWp ont été analysées. La moyenne annuelle de rayonnement solaire est de $3000 \mathrm{Wh} / \mathrm{m} 2 / j$ our ce qui implique une production électrique de $75.043 \mathrm{kWh} /$ an. Sur la base du prix de l'énergie solaire photovoltaïque régis par la Loi sur les Énergies Renouvelables (Erneuerbare Energien-Gesetz) existant en Allemagne et qui atteint un montant de $0,16 € / \mathrm{kWh}$, le projet devient viable lorsque le TIR (Taux Interne de Rentabilité) atteint 15,2\% et la VAN (Valeur Actualisée Nette) 14 966,72 $€$. 


\section{Mots-clés}

Énergies renouvelables, énergie solaire photovoltaïque, interconnexion électrique.

\section{Aplicação da energia solar fotovoltaica interligada à rede. Caso Thierhaupten estudo (Alemanha)}

\section{RESUMO}

Este estudo avalia a viabilidade de uma instalação solar fotovoltaica conectada à rede para geração de eletricidade em um ponto localizado em Thierhaupten, na cidade de Augsburg, no sul da Alemanha. O dimensionamento e projeto do sistema foram realizados utilizando o PVGIS, PV-Sol, Sol projeto, Würth e ferramentas de planilha Excel. Se analisa o desempenho técnico e econômico de um sistema composto de células fotovoltaicas e uma capacidade de 78,96 kWp. A média de radiação solar global média solar é de $3000 \mathrm{Wh} / \mathrm{m} 2$ / dia, e, assim se projeta uma geração de $75.043 \mathrm{kWh} /$ de eletricidade. Com base no preço de energia solar fotovoltaica, regida pelas regras da Lei de Energia Renovável (Erneuerbare Energien Gesetz), em vigor na Alemanha, no valor de $0,16 € / k W h$, os resultados permitiram concluir que o projeto estudado é viável com uma TIR (Taxa Interna de returno) de 15,2\% e VPL (Valor Presente Líquido) $d e € 14,966.72$.

\section{Palavras-chave}

Energia renovável, energia solar fotovoltaica, interligação elétrica. 


\section{Introducción}

TC a tecnología de las energías renovables es reconocida cada vez más como uno de los pilares centrales en el desarrollo de una estrategia energética sostenible (Oliver y Jackson, 1999), especialmente cuando es evidente que la demanda de energía continúa exponencialmente en incremento. En el caso de la Unión Europea (UE), se ha fijado la meta para 2020 de cumplir con el $20 \%$ de la demanda de energía, mediante el uso de fuentes renovables, donde la energía solar será sin duda una parte importante del futuro «mix» energético.

La razón principal de esta tendencia obedece a que el uso de la energía solar contribuye a la eficiencia de los países, considerando que se aprovechan sus propios potenciales para la generación de energía eléctrica y térmica, se reduce la emisión de gases de efecto invernadero, se disminuye la importación y utilización de los combustibles fósiles, y se promueve el desarrollo de la industria local mediante la generación de nuevos puestos de trabajo.

Al respecto, Alemania es líder en la instalación de paneles solares a gran escala sobre las cubiertas de industrias, hogares y granjas; donde la introducción de la Ley de Tarifas en 1999, dio lugar a un cambio significativo en las condiciones para los inversores de estos proyectos (Jager-Waldau, 2007). En 2013, la energía fotovoltaica generada ascendió a 30 TWh y cubrió aproximadamente el 5,7 por ciento del consumo neto de electricidad de Alemania (Wirth, 2014). Los sistemas fotovoltaicos se han concentrado en Baden-Württemberg y Augsburg, por su elevada irradiación solar (figura 1) y por contar con la mayor renta per cápita (Gorrozari, 2012). Por otro lado, la gran parte de las instalaciones fotovoltaicas se encuentran conectadas a la red eléctrica ( $91 \%$ del total) (Pingarrón, 2011).

Dadas estas características, el objetivo del presente trabajo es demostrar, mediante un caso aplicado, el proceso de diseño de una instalación fotovoltaica conectada a la red eléctrica, que será emplazada en la 
cubierta de una nave industrial en la localidad de Thierhaupten, en el estado de Augsburg; así como realizar la evaluación de factibilidad técnica y económica de acuerdo con la normatividad alemana.

Figura 1. Mapa de radiación solar de Alemania

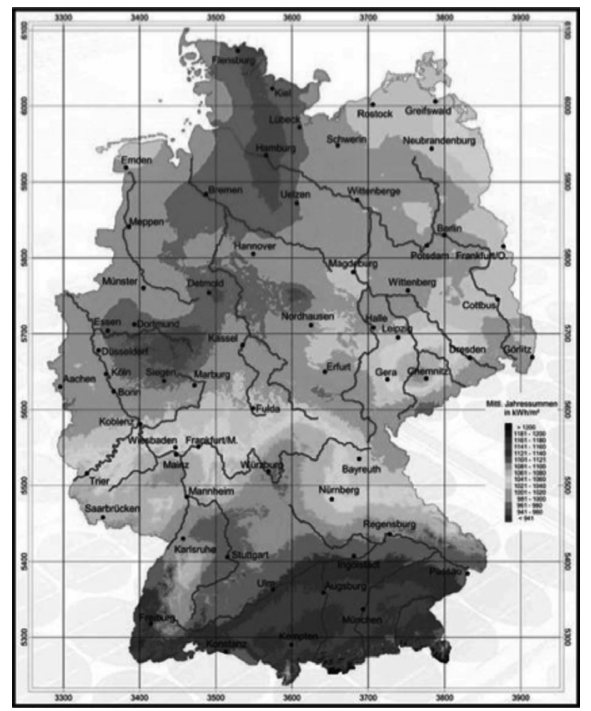

Fuente. Deutsches Wetteramt, 2011.

\section{Metodología}

Según el interés de la búsqueda de conocimiento, la metodología de la investigación es tipo aplicada, en la que se busca el desarrollo práctico del estudio en el marco de un proyecto o caso real (Vargas, 1999). Para dimensionar la instalación, se han usado programas informáticos existentes en el mercado y otros facilitados por las distintas casas comerciales. El proceso ha sido el siguiente:

- Evaluación de la disponibilidad del recurso haciendo uso del Software PVGis. 
- De forma simultánea se realiza la determinación del rendimiento específico de la instalación, empleando el programa PV-Sol; así como el dimensionado definitivo de la instalación, haciendo uso del Software Sunny Design, facilitado por la casa de inversores fotovoltaicos SMA.

- Una vez definido el número y modelo de los módulos y de los inversores, se determina la configuración de los Strings sobre la cubierta del edificio. Además, se establece teóricamente el número máximo de módulos por String.

- Utilizando el Software de la casa Würth, se calcula el sistema de montaje: tipo de sistema, puntos de anclaje, lista de material necesario, etc.

- Finalmente, se realiza el estudio de factibilidad económica de la instalación.

\section{Resultados}

\subsection{Localización del proyecto y recurso energético solar}

El presente proyecto para la instalación de células solares fotovoltaicas se realiza por solicitud de la empresa Ingenito\&Thumm GBR, dedicada a la fabricación de piezas para automóvil (figura 2). Toda la energía generada será para su venta a la red eléctrica alemana, por lo tanto, el sistema no requerirá baterías. La inversión inicial disponible para comprar 329 paneles, es alrededor de 120 mil euros. 
La empresa se encuentra ubicada en Am Unteren Stein 3, en la localidad de Thierphaupten (Alemania). La extensión de Thierhaupten es de $39,1 / \mathrm{km}^{2}$ y está situado a $470 \mathrm{~m}$ de altitud media sobre el nivel del mar. Sus coordenadas son $48^{\circ} 20^{\prime} \mathrm{N}-10^{\circ} 33^{\prime} \mathrm{E}$.

Figura 2. Nave industrial para emplazamiento del proyecto
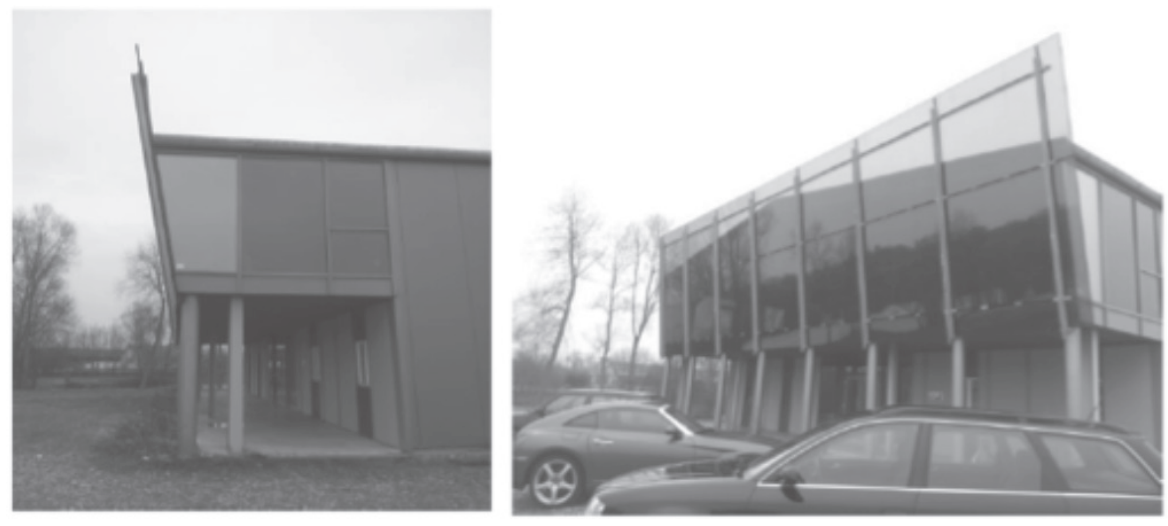

Fuente. Elaboración propia de los autores.

A continuación se presentan las características y dimensiones del edificio, las cuales son determinantes para la productividad y rentabilidad de la instalación (Tabla 1). Según el Software PV Gis, la máxima radiación se produce en el mes de julio y la mínima en el mes de enero, con 5310 y $888 \mathrm{Wh} / \mathrm{m}^{2} /$ día respectivamente, siendo la media anual de $3000 \mathrm{Wh} / \mathrm{m}^{2} /$ día. Las temperaturas medias a lo largo del día oscilan entre la máxima de $19.7^{\circ} \mathrm{C}$, registradas en julio y agosto, y las mínimas de $0.0^{\circ} \mathrm{C}$ registradas en el mes de enero, siendo la media de $10.3^{\circ} \mathrm{C}$.

\section{Tabla 1. Dimensiones del edificio}

\begin{tabular}{|l|l|}
\hline Alto & $6 \mathrm{~m}$. \\
\hline Largo & $42 \mathrm{~m}$. \\
\hline Ancho & $17,87 \mathrm{~m}$. \\
\hline Ancho cada vertiente & $9 \mathrm{~m}$. \\
\hline Angulo tej. & $7^{\circ}$ \\
\hline Orientación & $-5^{\circ}$ \\
\hline
\end{tabular}

Fuente. Elaboración propia de los autores. 


\subsection{Descripción técnica de la instalación}

\subsubsection{Determinación del rendimiento específico de la instalación}

Para analizar el comportamiento de los sistemas fotovoltaicos conectados a la red, se emplean tres parámetros diferentes: el índice de producción final del sistema (Yf), el índice de producción de referencia ( $\mathrm{Yr}$ ) y el performance ratio (PR) (Fernández, 2011). Se utiliza el Software PVSol en el cual se carga información de descripción del proyecto, datos climáticos como radiación sobre la horizontal, geometría del edificio, generalidades de los módulos, localización sobre cubierta, entre otros.

El índice de producción final del sistema (Yf) es la salida neta de energía dividida entre la potencia del campo de paneles. Representa el número de horas que el generador fotovoltaico necesitaría funcionar, a potencia nominal, para proporcionar la misma energía. Corresponde a un rendimiento específico anual y será determinante para evaluar la viabilidad económica del proyecto.

$$
Y f=\frac{E}{P 0}=950,4 k W h / k W p
$$

Este parámetro, es una normalización de la energía producida con respecto al tamaño del campo y es útil para comparar la energía producida por sistemas de diferente tamaño, aunque no lo es para identificar problemas de operación al no tener en cuenta la variabilidad de la radiación solar.

El índice de producción de referencia (Yr) es la irradiación total sobre el plano del generador $\left(\mathrm{H}\right.$ en $\left.\mathrm{kWh} / \mathrm{m}^{2}\right)$ dividida entre la irradiancia de referencia $(G)$. Yr, es el número de horas de Sol pico y define el recurso de radiación solar del sistema. Su resultado depende de la localización, orientación del campo y de la variación de la climatología.

$$
Y r=\frac{H}{G}=\frac{\frac{593525}{530,45}}{1}=1118,9 \text { Horas }
$$

El performance ratio (PR) es el cociente entre la productividad final (Yf) y la productividad de referencia (Yf). Es un parámetro adimensional, 
que expresa el efecto global de las pérdidas. Estas pérdidas, pueden ser debidas a problemas con los inversores, cableado, desacoplo, temperatura de módulos, suciedad, nieve, sombreados, etc. EI PR, representa una medida de lo bien dimensionada que está la instalación y lo que la producción se acerca a la producción ideal.

$$
P R=\frac{Y f}{Y r}=\frac{950,4}{1118,9}=85 \%
$$

\subsubsection{Dimensionado de los módulos fotovoltaicos}

La distribución de los módulos se realiza en un área total de $530 \mathrm{~m}^{2}$ sobre la cubierta. El diseño busca maximizar la producción anual de energía, teniendo en cuenta los principales parámetros que afectan al rendimiento de una instalación solar, como son la orientación, inclinación, sombras sobre los módulos fotovoltaicos y pérdidas inherentes en la fabricación de las células solares. Adicionalmente, el rendimiento del sistema será menor para paneles fijos, comparado con los que pueden ajustarse en términos del ángulo de inclinación u orientación, situación que ocurre cuando se tiene un sistema de seguimiento.

En este caso, tanto la orientación como la inclinación de los módulos son fijas, dado que van a ser las mismas que la de la cubierta sobre la cual se van a instalar. Por la orientación donde se ubica esta instalación (hemisferio Norte), al enfrentar los paneles hacia el rumbo Septentrional, resultaría en una pérdida de eficiencia de aproximadamente $40 \%$. Este porcentaje se reduce al acercamiento con la dirección Sur (Boxwell y Glasbey, 2012).

La inclinación óptima, si no va a (o no puede) modificarse a lo largo del año, para una ubicación de latitud $48.6^{\circ}$ correspondiente a la del proyecto, es de $40^{\circ}$. Este grado fue basado en los cálculos de Landau (2014) que explican que si la latitud es entre $25^{\circ}$ y $50^{\circ}$ se toma la latitud, se multiplica por 0,76 y se adicionan 3,1 grados. En las zonas que experimentan nevada fuerte, es aconsejable montar los paneles con una inclinación mayor para que la nieve caiga, especialmente si la demanda energética es alta durante los meses de invierno. Evidentemente, un panel con una 
cubierta de nieve no va a generar mucha potencia. Los meses de invierno en la localidad son suficientemente bajos en temperatura, por lo cual experimentarán la nieve.

Otras consideraciones importantes a la hora de decidir la inclinación, es la presencia de obstáculos que bloquean los rayos de sol y causan sombra. La producción caerá más rápidamente cuando, como en el caso presente, el material de los módulos es cristalino (Hankins, 2010).

El problema más grave relacionado con la sombra, es que no sólo disminuye el Output de un módulo sino que podría llegar a dañarse, incluso si la sombra bloquea tan sólo una célula del panel. Cuando existen algunas sombras, esta misma célula no produce corriente sino que la consume. Este consumo se alimenta de la generación de las demás células y se convierte a calor. A largo plazo se convertirá en un Hotspot y evitará el funcionamiento correcto del módulo entero. Para prevenir la posibilidad de los Hotspots y mantener un funcionamiento óptimo del sistema, se emplea un diodo de derivación (DGS, 2008). Sin embargo, para el presente caso no hay ninguna interferencia permanente, de maneraque una limpieza frecuente para quitar partículas de polvo, humedad, etc. será suficiente para minimizar su efecto.

Las pérdidas experimentadas, intrínsecamente en una célula solar, son las que derivan del reflejo, la eficiencia termodinámica, la recombinación y la resistencia eléctrica. Esta última tiene mucho que ver con el factor de forma y la temperatura (Castellano, 2010). El factor de formar representa el ratio de la potencia máxima divida por el voltaje en el circuito abierto (Vca) y la corriente del corto circuito (Icc). Este factor para los paneles cristalinos tiene un valor típico de entre 0,7 y 0,85 (Moro, 2010).

Vale la pena decir que un incremento en temperatura disminuirá el rendimiento del módulo solar (Ehrlich, 2013) y por lo tanto, la ventilación natural entre los módulos, es una consideración importante para el inversor, al momento de diseñar una instalación fotovoltaica.

$$
F F=\frac{P_{m}}{V_{o c} \times I_{s c}}=\frac{\eta \times A_{c} \times E}{V_{c c} \times I_{s c}}
$$


También, debe recordase que la potencia pico del generador fotovoltaico que proporciona el fabricante, se obtiene a $25^{\circ} \mathrm{C}$. Para tener estas temperaturas de célula, la temperatura exterior deberá ser de entre $-10^{\circ} \mathrm{C}$ y $0^{\circ} \mathrm{C}$, lo que podría ocurrir naturalmente en Thierhaupten durante los meses de diciembre o enero, o tal vez algunos días de noviembre o febrero.

Ahora bien, el Software empleado para realizar el diseño de la instalación consiste en el Sunny Design de la compañía SMA. Este es un Software profesional de acceso gratuito, es ampliamente utilizado para diseño de instalaciones fotovoltaicas conectadas a la red y considera la normatividad alemana para sus cálculos; sin embargo, presenta restricciones en cuanto a la disponibilidad de bases de datos de inversores debido a que se limita a los ofertados por la casa manufacturera (Paraskevadaki 2014; Photovoltaic Software 2014).

Una vez ingresada la información de base en el Software, los módulos seleccionados para el proyecto son los Bull PowerTech P6/240, con una potencia de $240 \mathrm{~W}$. La tabla 2 presenta las demás características técnicas de los módulos. Por la configuración del área, se estableció el número óptimo de módulos en un valor de 329 , por lo tanto, la potencia total instalada asciende a $78,96 \mathrm{kWp}$. Del total de unidades, 189 conforman el sistema fotovoltaico 1 , cuya orientación es de $-5^{\circ}(\mathrm{SE})$, lo cual significa, según Boxwell y Glasbey (2012), una pérdida de 10\% comparado con la posición óptima. Los restantes 140 , forman el sistema 2 con una orientación de $175^{\circ}(\mathrm{NO})$. La pérdida registrada en este caso sería alta con un valor al alrededor de $30 \%$. Estas dos orientaciones ocurren por el hecho de que la cubierta es a dos aguas. En el proyecto presente, la inclinación por petición del promotor de la instalación será la propia de la cubierta, es decir $7^{\circ}$. Ambas, la inclinación y la orientación, tienen que ver con la posición de la propia cubierta.

Aunque, ni la inclinación ni la orientación son las óptimas para obtener la máxima producción de energía, se han establecido con el fin de reducir los costos de instalación y montaje, (tanto de mano de obra como de material), aunque ello pudiera penalizar la producción y la rentabilidad futuras. 
Tabla 2. Características técnicas de los módulos Bull PowerTech P6/240

\begin{tabular}{|c|c|c|c|}
\hline Pmax & $240 W$ & Ef. Celda & $17 \%$ \\
\hline Vmp & $29,9 \mathrm{~V}$ & Ef. mód. & $14,9 \%$ \\
\hline Imp & $8,0 \mathrm{~A}$ & Coef. Ta (Pmax) & $-0,45 \% /{ }^{\circ} \mathrm{C}$ \\
\hline Isc & $8,5 \mathrm{~A}$ & Coef. Ta (Voc) & $-0,35 \% /{ }^{\circ} \mathrm{C}$ \\
\hline Voc & $37,4 \mathrm{~V}$ & Coef. Ta (Isc) & $+0,04 \% /{ }^{\circ} \mathrm{C}$ \\
\hline
\end{tabular}

Fuente. Elaboración propia de los autores.

Con el fin de incrementar la productividad de la instalación, los autores se han elegido una configuración de generador particionado, esto quiere decir, que se conecta más de un String a cada inversor.

\subsubsection{Dimensionado inversores fotovoltaicos}

Los inversores son los dispositivos que convierten la corriente continua producida por los módulos fotovoltaicos a la corriente alterna utilizada por la red eléctrica, en el caso de On-Grid, o almacenada en baterías, en el caso de Off-Grid. En el presente proyecto, toda la energía generada por parte del sistema fotovoltaico (FV) se alimentará la red eléctrica alemana.

La potencia de los inversores es la que determina la potencia nominal de la instalación, mientras su posicionamiento dependerá sobre la cubierta del edificio (Vallina, 2010). Los valores de potencia del generador FV y del inversor deben corresponderse óptimamente entre sí. Si la instalación fotovoltaica es demasiado grande, se perderá energía debido a la función limitadora de corriente en condiciones de sobrecarga del inversor. En los casos, como en este proyecto, en que el sistema fotovoltaico no está orientado óptimamente o sombreado parcialmente, es recomendable (tanto desde el punto de vista técnico como económico) realizar el dimensionado del inversor con una menor potencia. La potencia nominal del inversor puede ser entre un $10 \%$ y un $20 \%$ inferior a la potencia del generador fotovoltaico, dado que la potencia pico del generador se alcanza en situaciones puntuales y por lo tanto, el inversor suele trabajar en valores del $70 \%$ de su potencia máxima (Santamaría y Castejón, 2010). También hay que recordar la existencia de pérdidas de potencia por desacoples de módulos, caídas de tensión en los conductores y por la degradación de los módulos con el paso del tiempo. 
Por esta razón, al subdimensionar el inversor se logran mantener niveles de eficiencia aceptables, incluso para bajos niveles de irradiación. Dicho esto, un subdimensionado puede implicar una sobrecarga del inversor, por lo que se debe conocer claramente su funcionamiento en caso de sobrecarga y configurar el generador fotovoltaico, de tal manera que se evite esta posibilidad (Rubio, 2007).

Adicionalmente, la reciente tecnología permite contar con inversores multi-Input (multi-strings), lo que significa que se puede dividir la potencia de forma proporcional al número de entradas, de forma que el seguimiento al punto de máxima potencia lo realiza de forma independiente.

Mediante el Software Sunny Design se seleccionaron cinco (5) unidades de inversores con referencia SMA SunnyTriPower 15000TL-10 (tabla 3).

Tabla 3. Características técnicas de los inversores SMA SunnyTriPower 15000TL-10.

\begin{tabular}{|l|l|}
\hline Potencia máxima de CC & $15340 \mathrm{~W}$ \\
\hline Tensión máxima CC & $1000 \mathrm{~V}$ \\
\hline Rango de tensión MPP & $360-800 \mathrm{~V}$ \\
\hline Tensión nominal de CC & $600 \mathrm{~V}$ \\
\hline Tensión de CC min/ Tensión inicial & $150-188 \mathrm{~V}$ \\
\hline Corriente max. de entrada/ por string & $\mathrm{A}: 33 \mathrm{~A}$ B: 11 A / 33 A \\
\hline Potencia nominal CA & $15000 \mathrm{~W}$ \\
\hline
\end{tabular}

Fuente. Elaboración propia de los autores.

La verificación del inversor seleccionado dependerá de la configuración de Strings, tal como se muestra a continuación, partiendo del número de módulos que se instalarán en la cubierta debido al área disponible de la cubierta.

\subsubsection{Configuración de Strings}

Según Maeda (2011) en una instalación Off-Grid no es habitual tener más que un módulo por cada String, sin embargo, para un sistema que alimente la red eléctrica es necesario conectar muchos paneles en 
serie para generar una tensión DC suficientemente alta que pueda ser compatible con el sistema AC.

El número de paneles conectados en serie y en paralelo determinará la tensión y corriente experimentada, respectivamente por el inversor. De los dos, el voltaje es el más importante. Por esta razón, cuando se quiere establecer la mejor configuración del sistema solar, deben considerarse dos umbrales: la tensión mínima y máxima. Con el control de la primera se previenen las averías del inversor por tener demasiada tensión, mientras que con la segunda se asegura que el mismo empezará a funcionar en el día más caluroso del año (cuando la tensión es la más baja). El número óptimo de paneles que forman un String, es decir conectados en serie, dependerá también de varios factores incluyendo las temperaturas máximas y mínimas experimentadas por la ubicación, el rango de operación para el inversor, el cable y si los módulos funcionan en aislamiento (con baterías) o están conectados a la red eléctrica. Igualmente, es importante tener en cuenta, que para evitar el problema de Mismatch, solamente deben ser conectados en serie al mismo String módulos con las mismas características (el mismo tipo/marca) y bajo condiciones similares de orientación y sombreamiento (Farrett y Godoy, 2006). Esta consideración es importante porque el panel que experimenta la menor radiación, determina la corriente de todos. Cuando una sombra parcial es inevitable, a veces es mejor instalar varios inversores para mejor el rendimiento.

La máxima tensión de salida del generador fotovoltaico debe ser inferior que la máxima tensión de entrada DC del inversor (Braun et al, 2012). Dado que la máxima tensión del sistema es la que se obtiene cuando funciona en circuito abierto a bajas temperaturas, el umbral máximo de paneles conectados en serie se establece en el día más frío del año. En el presente caso, para minimizar riesgos, los autores han seleccionado una temperatura mínima de $-10^{\circ} \mathrm{C}$, de esta forma, se puede determinar el número máximo de módulos a instalar en serie, en un String en paralelo, mediante la siguiente fórmula:

$$
N \operatorname{smax}=\frac{\operatorname{Umax}(\text { inversor })}{U \operatorname{Ua}\left(\operatorname{modulo} a-10^{\circ} \mathrm{C}\right)}
$$


Respecto a la cantidad mínima de módulos por String, debido a las altas temperaturas que se pueden alcanzar en verano en Thierhaupten, un módulo fotovoltaico podría registrar una tensión inferior a la obtenida bajo condiciones Standard Test Conditions (STC). Cuando es así, la tensión de trabajo del sistema disminuirá por debajo de la mínima tensión de seguimiento o del punto de máxima potencia del inversor y este no alcanzará a inyectar la máxima cantidad posible de potencia, o incluso se podría apagar o no iniciar. Para determinar el número mínimo de módulos en serie se utiliza la siguiente ecuación:

$$
N \min =\frac{U \min (P M P \text { inversor })}{U m p p\left(\text { modulo } a{ }^{\circ}{ }^{\circ} C\right)}
$$

Por la forma de la cubierta, el proyecto requiere Strings independientes para aprovechar el espacio disponible (y optimizar la generación eléctrica). Esta configuración permite, considerando que es necesario, diferencias en el número de paneles conectados en serie y sus orientaciones. Si un módulo se encuentra más sombreado o con un menor nivel de radiación que otro, no se afecta el desempeño del mejor ubicado (Boxwell, 2012). En esta instalación cada String tiene su propio inversor.

Se presenta la configuración en cuanto al número de Strings y de módulos por cada String por instalación (Tabla 4).

Tabla 4. Configuración de la instalación.

\begin{tabular}{|l|l|}
\hline GENERADOR FV 1 & \\
\hline $\mathrm{N}^{\circ}$ de módulos & 189 \\
\hline $\mathrm{N}^{\circ}$ de inversores & 3 \\
\hline Mod. Inversores & STP 15000LT-10 \\
\hline $\mathrm{N}^{\circ}$ de Strings y No módulos por String & $2 \times 22 / 1 \times 19$ \\
\hline GENERADOR FV 2 & \\
\hline $\mathrm{N}^{\circ}$ de módulos & 140 \\
\hline $\mathrm{N}^{\circ}$ de inversores & 2 \\
\hline Mod. Inversores & STP $15000 \mathrm{LT}-10$ \\
\hline $\mathrm{N}^{\circ}$ de Strings y $\mathrm{N}^{\circ}$ módulos por String & $3 \times 21 / 1 \times 7$ \\
\hline
\end{tabular}

Fuente. Elaboración propia de los autores. 


\subsubsection{Dimensionado del cableado}

Los módulos solares producen energía en DC, así mismo, todos los cables deben ser resistentes a altas temperatura y las condiciones climáticas que se encuentran en la ubicación. Específicamente, para el sistema DC los cables deben ser los suficientemente largos para construir los Strings y conectarlos a través de una caja de reagrupación a los inversores. El tamaño típico para un cable cobre de DC es de 4 a $6 \mathrm{~mm}$, es decir, 10 o 12 AWG. Al final de cada uno de los Strings existe otro cable para minimizar las pérdidas eléctricas, siendo normalmente iguales o un poco más grande. La pérdida eléctrica en términos de tensión, desde la caja de agrupación al inversor central puede ser considerable. La máxima pérdida permisible no puede ser mayor que el $2 \%$ (Fong y Tipett, 2012). La caída de tensión $\Delta \mathrm{V}$ a lo largo de un conductor de longitud $L(\mathrm{~m})$, sección $\mathrm{S}$ $\left(\mathrm{mm}^{2}\right)$ y conductividad $\mathrm{y}\left(\mathrm{S} . \mathrm{m} / \mathrm{mm}^{2}\right)$, se calcula de la siguiente manera:

$$
\Delta V=\frac{1}{r} * \frac{L}{S} * I
$$

Para el caso de la presente instalación, se toma la longitud máxima, es decir, la longitud del cable que conecta el módulo más alejado de inversores, los cuales se instalarán en la esquina noreste del edificio.

La longitud total aproximada del citado cable, será la longitud de la nave más el ancho de cada una de las vertientes, más la distancia que hay desde la cubierta hasta donde se colocará. Los inversores se colocarán a $2 \mathrm{~m}$ del suelo aproximadamente, por lo tanto, esta distancia será de $2 \mathrm{~m}$ unos $4 \mathrm{~m}$. Con lo cual, la longitud del cable es de $64 \mathrm{~m}$.

Puesto que el cable posee recorrido de ida y vuelta, se calculará la caída de tensión utilizando el doble de dicha distancia.

El String más alejado de los inversores, está compuesto por 22 módulos en serie, con una potencia pico de $5,28 \mathrm{kWp}$ por String. La máxima intensidad que circulará por dicho conductor, corresponderá a la del punto de máxima potencia cuando la tensión es mínima, es decir, $\mathrm{V}_{\mathrm{mpp}}$ $\left(\mathrm{T}=70^{\circ} \mathrm{C}\right)$. 


$$
\operatorname{Imax}=\frac{5,28}{22} * 34,6=8,30 \mathrm{~A}
$$

La conductividad del material, depende de la agrupación de cables, el método de instalación y la temperatura a la que el mismo está sometido (DGS, 2008). Para cables fabricados en cobre, esta toma valores de 56 a $20^{\circ} \mathrm{C}$ y 48 a $70^{\circ} \mathrm{C}$ (Santamaría y Castejón, 2009; López, 2004). En caso de trabajar a $70^{\circ} \mathrm{C}$, temperatura que puede experimentar una teja y por lo tanto es una consideración importante para el presente caso, la caída de tensión de un cable con una superficie de $4 \mathrm{~mm}^{2}$ está definida por el siguiente resultado:

$$
\Delta V=\frac{1}{48} * \frac{2 * 64}{4} * 8,3=5,53 V
$$

Al expresar la caída de tensión en porcentaje, se obtiene:

$$
\Delta V=\frac{5,53}{528} * 1=1,047 \%
$$

Valor que se encuentra dentro del máximo recomendado de $2 \%$. Para reducir las pérdidas, se podría optar por instalar un cable de $6 \mathrm{~mm}^{2}$ en lugar de $4 \mathrm{~mm}^{2}$, sin embargo se encarecerían los conductores. De esta forma, se podría reducir la caída de tensión a un $0,69 \%$.

Para el caso de los conductores de la línea AC de salida de los inversores, es importante determinar la distancia al punto en el cual la instalación se conectará a la red, información que es provista por la compañía eléctrica. También debe calcularse la potencia pico ingresada a cada una de las fases. La tensión de salida de línea será de $230 \mathrm{~V}$ y la potencia ingresada a cada fase se calcula como la suma de las potencias nominales de los inversores dividida entre tres. La corriente circulante por cada fase será:

$$
I=\frac{5 * 15000}{3 * 230}=108,69 A
$$

En el presente caso, la distancia de los inversores a los contadores de la compañía eléctrica será de unos $30 \mathrm{~m}$. Para los cálculos, se establece 
una temperatura de los conductores de $20^{\circ} \mathrm{C}$ (aunque no siempre se podrá cumplir con esta premisa, especialmente en verano) y se asumen unas pérdidas inferiores al $2 \%(4,62 \mathrm{~V})$. Con estas especificaciones, la superficie mínima de cada fase será:

$$
S \geq \frac{1}{56} * \frac{30}{3,45} * 108,69 \geq 16,87 \mathrm{~mm} 2
$$

Usando tamaño de cable de 4 AWG (American Wire Gauge) por cada fase, lo cual es equivalente a $21,15 \mathrm{~mm} 2$ y se obtienen pérdidas del $1,2 \%$. Al igual que para el cableado de DC, se podrían reducir las pérdidas aumentando la sección del cable, aunque se incrementarían los costes.

\subsubsection{Dimensionado del sistema de montaje}

Las funciones del sistema de montaje o estructura soporte, son aquéllas que proporcionan soporte y sujeción segura a los módulos, facilitando su instalación, así como dotan a los módulos de la inclinación y orientación adecuadas (o deseadas), evitando también sombras.

Las principales características y/o recomendaciones de la estructura de montaje son:

- Debe ser apta para su instalación a la intemperie, protegida contra la corrosión. Los materiales más comunes son el acero galvanizado, acero y aluminio.

- Debe estar bien anclada, para soportar las cargas máximas producidas por el viento. Debe, además, permitir dilataciones térmicas.

- Los puntos de sujeción de los módulos deben ser los suficientes para que no se produzcan cargas excesivas.

- El diseño de la estructura debe permitir la reparación y recambio de módulos, así como su limpieza en caso de ser necesaria. 
- Debe disponer de toma de tierra.

- La tornillería debe ser de acero inoxidable. En el caso de ser la estructura galvanizada, podrán ser tornillos galvanizados. La sujeción de los módulos a la estructura será mediante tornillería de acero inoxidable.

Para decidir la tipología de estructura de montaje, también hay que considerar el tipo de cubierta disponible. En el caso del presente proyecto, se trata de una cubierta metálica de panel sándwich, formada por paneles prefabricados, compuestos por una chapa interior, un aislante intermedio de espuma de poliuretano o lana de roca y una chapa exterior.

Para este tipo de techos, existen muchos sistemas de fijación de paneles. Algunos fijan rieles de aluminio a lo largo del mismo para fijar los paneles, mientras que otros consisten en segmentos cortos de metal que se fijan al techo y permiten el montaje de los paneles. Hay sistemas que se fijan mediante largos tornillos especiales a las vugas que forman la estructura; que están debajo de las láminas y otros que se fijan a las láminas directamente, pero se requiere de un espesor de lámina mínimo, a fin de que el sistema pueda soportar los esfuerzos a los que está sometido. Estos sistemas, además de ser más fáciles y rápidos de instalar, presentan la ventaja de no depender de la estructura del edificio, ni del material de las vigas, de su espesor o de la distancia entre ellas.

El sistema de montaje elegido es el de segmentos cortos (de esta forma no será necesario junta de dilatación). En este caso, los materiales usados serán de la casa Würth, por lo cual, para el dimensionado de la estructura se utiliza el Software que dicha casa dispone para determinar las cantidades necesarias de cada uno de los materiales.

\subsection{Análisis financiero}

La normativa de aplicación en Alemania en cuanto a instalaciones fotovoltaicas, así como su remuneración, consiste en la Ley de Energías Renovables o Erneuerbare Energien Gesetz, (EEG por sus siglas en alemán). A los productores se les garantiza una remuneración 
correspondiente al año de instalación, constante durante 20 años, que será estipulada por la EEG vigente en el momento de la construcción (Pingarrón, 2011). En todo caso, los incentivos a la inversión para la generación de energía fotovoltaica no son apoyados por fondos públicos, por lo que el sistema no contará con subsidios para su instalación (Wirth, 2014). Para determinar la viabilidad de la inversión, se manejarán los indicadores de Valor Presente Neto (VPN) y Tasa Interna de Retorno o Rentabilidad (TIR), Ambos tienen en cuenta el flujo de caja a lo largo de la vida del proyecto (Aranda y Scapellini, 2009).

De acuerdo con IRENA (2012) para instalaciones fotovoltaicas de más más de $10 \mathrm{kWp}$, los costos de instalación pueden variar entre $1500 \mathrm{y}$ $2700 € / \mathrm{kWp}$. De los cuales, en una instalación sobre tejado, normalmente los costos se distribuyen en un $50 \%$ para los módulos y la mitad restante utilizada para estructura y estanterías, preparación del sitio, instalación eléctrica, inversor y administrativos. Tal como se mencionó, no se requiere inversión en dispositivo de almacenamiento. Por su parte, la generación eléctrica fotovoltaica alcanzó valores de costo de generación de energía eléctrica (LCOE) entre 0,078 y 0,142 €/kWh (Kost et al, 2013) respecto a una precio esperado de venta de $0,1694 € / \mathrm{kWh}$.

A continación, se observan los resultados de VPN y TIR del proyecto con una vida útil de 20 años (Tabla 5):

Tabla 5. Resultados económicos del proyecto.

\begin{tabular}{|l|l|}
\hline Inversión inicial & $€ 119,153.75$ \\
\hline Venta de electricidad & $€ / \mathrm{kWh} 0.1694$ \\
\hline Costos y gastos & $€ / \mathrm{kW} \mathrm{20}$ \\
\hline LCOE & $€ / \mathrm{kWh} 0.140$ \\
\hline TIR & $15.2 \%$ \\
\hline VNA & $€ 14,966.72$ \\
\hline Payback time & 6 \\
\hline
\end{tabular}

Fuente. Elaboración propia de los autores. 
Un análisis de sensibilidad fue desarrollado con el fin de medir el impacto de la inversión inicial frente a la rentabilidad del proyecto. Se muestra el resultado de la evaluación frente a una tasa de oportunidad del $8 \%$ (Figura $3)$. Es importante señalar que Alemania y China cuentan con los precios de venta de tecnología solar fotovoltaica más bajos del mercado a nivel mundial, sin embargo, a medida que evoluciona la norma de regulación eléctrica, los márgenes de utilidad se ven reducidos cada vez más.

Figura 3. Análisis de sensibilidad de la rentabilidad del proyecto (TIR) vs. inversión inicial.

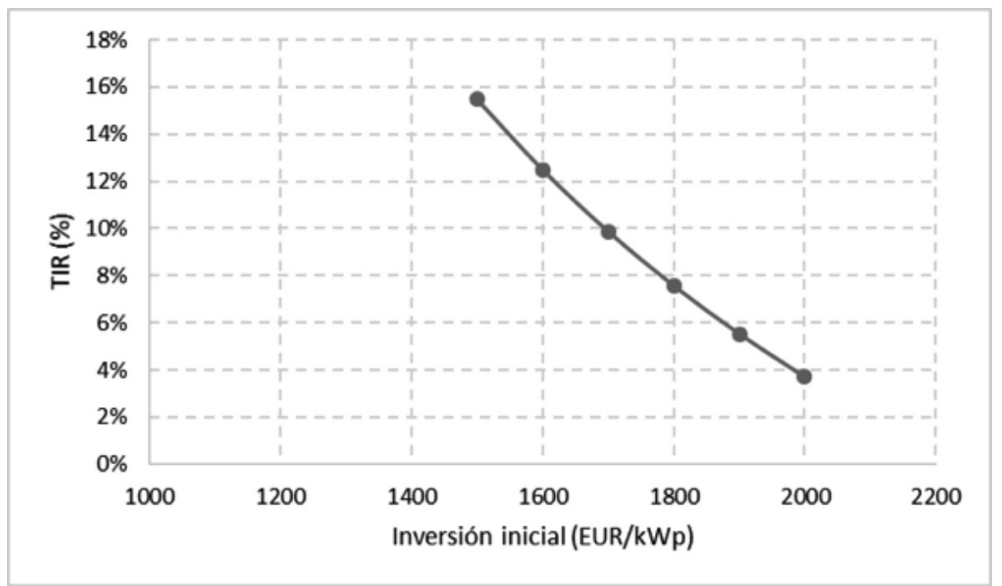

Fuente. Elaboración propia de los autores.

Otro beneficio a cuantificar con el proyecto, es la posibilidad de ahorros por la contaminación evitada o mitigada, al compararla la tecnología fotovoltaica con los combustibles convencionales en términos de emisiones de dióxido de carbono $\left(\mathrm{CO}_{2}\right)$ hacia la atmósfera. Según Gerhlicher Solar (2014), la reducción de $\mathrm{CO}_{2}$ es 700 a 800 kg por cada kWp. Dado que el proyecto es de $78,96 \mathrm{kWp}$, el ahorro promedio representa aproximadamente 62250 toneladas anuales de $\mathrm{CO}_{2}$. Adicionalmente, la opción de reducir impuestos por reducir los gases de efecto invernadero y la consideración de otros costos indirectos por no hacer nada para combatir el cambio climático, pudieran incluso hacer la inversión aún más atractiva desde las perspectivas tanto económica como ambiental. 


\section{Conclusiones}

El presente estudio examina la viabilidad de generación de electricidad mediante una instalación solar fotovoltaica interconectada a la red eléctrica, en un emplazamiento ubicado al Sur de Alemania. Para ello, se han utilizado diversos programas de dimensionado, tanto de uso privado como gratuitos, proporcionados por las diferentes casas comerciales. Se determinaron el número de módulos e inversores, así como la configuración de los Strings sobre la cubierta del edificio y el sistema de montaje a ejecutar. Se analizó el rendimiento técnico y económico de un sistema con potencia de $78,96 \mathrm{kWp}$. Se evaluó que este puede generar $75.043 \mathrm{kWh}$ de electricidad anual a coste normalizado de generación de energía alrededor de 0,14 €/kWh. Adicionalmente, se observó que al incrementar los costos iniciales de instalación (módulos, inversores, instalaciones, etc.), la rentabilidad del proyecto puede desmejorar significativamente. Además, se identificó que la radiación solar es determinante para establecer la productividad de este sistema. Se espera que la incorporación de la energía solar fotovoltaica a la red pueda reducir la generación de gases efecto invernadero y otras emisiones contaminantes asociadas a la generación de electricidad.

El presente caso puede servir como insumo para el desarrollo real por parte de la empresa objeto de estudio. Con base en los hallazgos de este estudio, se determina que la instalación fotovoltaica conectada a la red en Thierhaupten es técnica y económicamente viable.

Igualmente el artículo demuestra la viabilidad técnica y financiera de esta energía en Colombia, un país con un promedio de radiación solar de 4500 $\mathrm{Wh} / \mathrm{m} 2 /$ día con regiones, como La Guajira que cuentan con $6000 \mathrm{Wh} / \mathrm{m} 2 /$ día (UPME, 2005). Esta es mucho mayor que la cantidad registrada en Alemania y por lo tanto podría resultar en una mayor ganancia para un proyecto ubicado en zonas muy insoladas. 


\section{Referencias bibliográficas}

Aranda A., Scarpellini, S. (2009). Análisis de viabilidad económicofinanciero de un proyecto de energías renovables. España: Prensas Universitarias de Zaragoza.

Boxwell, M. y Glabey, S. (2012). Solar Electricity Handbook: A Simple, Practical Guide to Solar Energy: how to Design and Install Photovoltaic Solar Electric Systems. Greenstream Publishing. pp 197.

Braun, H. Banavar, M. y Spanias, A. (2012). Signal Processing for Solar Array Monitoring, Fault Detection, and Optimization (Google eBook). Morgan \& Claypool Publishers

Castellano, R. (2010). Solar Panel Processing. Archives contemporaines

Ehrlich, R. (2013). Renewable Energy: A First Course. CRC Press.

Farret F.A., Godoy, M. (2006). Integration of Alternative Sources of Energy. John Wiley \& Sons.

Gerlicher Solar. (2014). How Much Carbon Dioxide do I save with a PV System. Recuperado de http://www.gehrlicher.com/br/servico/faq/ how-much-co2-do-i-save-with-a-pv-system/

Gorozarri, B. (2012). Estudio de mercado energías renovables en Alemania (fotovoltaica, eólica y biomasa). España. Comunidad de Madrid.

Hankins, M. (2010). Stand-alone Solar Electric Systems: The Earthscan Expert Handbook for Planning, Design and Installation (Google eBook). Routledge.

Jager-Waldau, A. (2007). Photovoltaics and renewable energies in Europe.

Renewable and Sustainable Energy Reviews 11. pp1414-1437

López, R. (2004). Nuevo método de cálculo de secciones de cables de baja tensión. Recuperado de: http://www.tecnicaindustrial.es/tiadmin/ numeros/11/39/a39.pdf p37. 
Moro, M. (2010). Instalaciones solares fotovoltaicas (Google eBook). Editorial Paraninfo.

Oliver, M., Jackson, T. (1999). The market for solar photovoltaics. Energy Policy 27, pp 371-385

Paraskevadaki, E. (2014). Design a $500 \mathrm{~kW}$ solar project with SMA's Sunny Design - Location Turkey. Recuperado de: http://www.intosolar.com/2014/01/sunny-design-self-consumption-turkey.html

Pingarrón, M. (2011). El sector de la energía solar fotovoltaica en Alemania. España: Oficina Económica y Comercial de la Embajada de España en Berlín. Instituto Español de Comercio Exterior.

Photovoltaic Software. (2014). Inverters softwares for photovoltaic (PV) system design Recuperado de: http://photovoltaic-software.com/ inverters.php

Rubio, O. (2007). Diseño y Construcción de un Parque Fotovoltaico de 2MW en Albesa. España: Universitat Politécnica de Catalunya

Santamaría, G. y Castejón, A. (2009). Electrotécnia. Editex. p. 295. (2010). Instalaciones solares fotovoltaicas. Editex.

UMPE. (2005). Atlas de Radiación Solar de Colombia. Recuperado de http://www.upme.gov.co/Docs/Atlas_Radiacion_Solar/1-Atlas_ Radiacion_Solar.pdf

Vargas, A. (1999). Metodología de la Investigación. México: Spanta.

Wirth, H. (2014). Recent Facts about Photovoltaics in Germany. Germany: Fraunhofer Institut for Solar Energy Systems ISE - Fraunhofer ISE. 


\section{Bibliografía}

Deutsches Wetteramt, (2011). Radiación solar en Alemania. Recuperado de: http://www.zonu.com/detail/2011-05-25-13764/Radiacion-solaren-Alemania.html

Deutsche Gesellschaft für Sonnenenergie (DGS). (2008). Planning and Installing Photovoltaic Systems: A Guide for Installers, Architects and Engineers. Earthscan.

Fernández, O. (2011). Análisis de producción y rentabilidad de proyectos fotovoltaicos. Proyecto fin de carrera. Escuela Superior de Ingeniero de Sevilla.

Fong, A. y Tippett, J. (2012). Project Development in the Solar Industry. CRC Press.

International Renewable Energy Agency (IRENA). (2012). Solar Photovoltaics. Renewable energy technologies: cost analysis series, 1: Power Sector, Issue 4/5.

Kost, C., Mayer, J.N., Thomsen, J., et al (2013). Levelized cost of electricity renewable energy technologies. Germany: Fraunhofer Institut For Solar Energy Systems ISE - Fraunhofer ISE.

Landau, C. (2014). Optimum Tilt of Solar Panels. Recuperado de: http:// www.solarpaneltilt.com/

Maeda, M. (2011). How to Solar Power Your Home: Everything You Need to Know Explained Simply (Google eBook). Atlantic Publishing Company.

Photovoltaic Software. (2014) Inverters Softwares for photovoltaic (PV) system design. Recuperado de: http://photovoltaic-Software.com/ inverters.php 
